\title{
Treatment of secondary hyperparathyroidism: the clinical utility of etelcalcetide
}

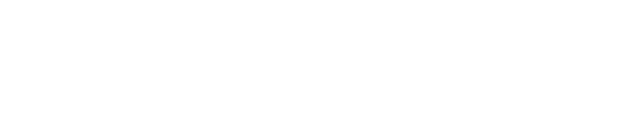

\section{Mario Cozzolino' \\ Andrea Galassi' \\ Ferruccio Conte' \\ Michela Mangano' \\ Luca Di Lullo² \\ Antonio Bellasi ${ }^{1,3}$}

'Department of Health Sciences, Renal Division, University of Milan, ASST Santi Paolo e Carlo, San Paolo Hospital, Milan, ${ }^{2}$ U.O.C. Nefrologia e Dialisi, Ospedale L. Parodi Delfino, Colleferro, Roma, ${ }^{3}$ Sant'Anna Hospital, ASST-Lariana, Como, Italy
Correspondence: Mario Cozzolino Department of Health Sciences, Renal Division, University of Milan, School of Medicine, San Paolo Hospital, Via di Rudini, 8 - 20I42, Milan, Italy Tel +39028184 438I

Email mario.cozzolino@unimi.it
Abstract: Secondary hyperparathyroidism (SHPT), a very frequent, severe, and worsening complication of chronic kidney disease, is characterized by high serum parathyroid hormone (PTH), parathyroid gland hyperplasia, and disturbances in mineral metabolism. Clinically, SHPT shows renal osteodystrophy, vascular calcification, cardiovascular damage, and fatal outcome. Calcium-sensing receptor (CaSR) is the main physiological regulator of PTH secretion; its activation by calcium rapidly inhibits PTH. Another important player in regulating mineral metabolism is vitamin D receptor (VDR), which is under the influence of vitamin D and influences the intestinal absorption of calcium and phosphate, PTH gene expression, and bone calcium mobilization. Serum phosphate levels influence fibroblast growth factor 23 (FGF-23) production, a phosphatonin that modulates serum phosphate reabsorption, PTH synthesis, and vitamin D production. Current therapeutic approaches consist of 1) phosphate intake control by diet or phosphate binders, 2) vitamin D by VDR activation, and 3) calcimimetic agents that activate CaSR. Recently, a new long-acting peptide (etelcalcetide) belonging to the calcimimetics class was approved for intravenous use in hemodialysis patients with SHPT. Etelcalcetide binds directly to CaSR, by a sulfide bond, inhibiting the production and secretion of PTH by parathyroid glands. After intravenous administration in rats, etelcalcetide is quickly distributed to the tissues and eliminated by kidneys, while in uremic animals the nonrenal excretion is only $1.2 \%$. In hemodialysis patients, the treatment itself is the main route of elimination. Etelcalcetide in hemodialysis patients with SHPT was more effective than placebo and cinacalcet, with a PTH reduction of $>30 \%$ in $76 \%$ of patients with etelcalcetide versus $10 \%$ with placebo. Particular attention was paid to the safety of the drug; the most common adverse event was asymptomatic blood calcium reduction, similar to cinacalcet, while gastrointestinal symptoms were less frequent. This promising new drug available for better control of SHPT will, together with drugs already in use, optimize the treatment to normalize the biochemical parameters.

Keywords: secondary hyperparathyroidism, CKD-MBD, calcimimetic, dialysis

\section{Pathogenesis of secondary hyperparathyroidism in chronic kidney disease}

Parathyroid hormone (PTH) is a key regulator of calcium metabolism; it stimulates bone reabsorption, increases serum calcium and phosphorus levels, and promotes vitamin D synthesis. ${ }^{1}$ Secondary hyperparathyroidism (SHPT), a common, serious, and progressive complication of chronic kidney disease (CKD), is characterized by high serum PTH, parathyroid gland hyperplasia, and disturbances in mineral metabolism, mainly hypocalcemia and hyperphosphatemia. ${ }^{1}$ These mineral disturbances mainly cause renal osteodystrophy, progressive vascular calcification, and in turn, cardiovascular disease and death, especially in patients receiving hemodialysis. ${ }^{2-5}$ Together, this constellation of comorbidities is known as CKD-mineral and bone disorder (CKD-MBD). ${ }^{6}$ 
In the US, the estimated prevalence of SHPT in patients with CKD ranges from 2 to nearly 5 million individuals, with $30 \%-50 \%$ of end-stage renal disease (ESRD) patients affected by SHPT. ${ }^{3}$

Under normal physiological conditions (Figure 1), the principal regulator of PTH secretion and parathyroid gland function is the calcium-sensing receptor (CaSR). ${ }^{4-7}$ Activation of the CaSR by serum calcium rapidly inhibits PTH synthesis and secretion and parathyroid gland growth. Furthermore, the CaSR influences PTH gene expression and may also upregulate the vitamin D receptor (VDR). Parathyroid glands express high levels of the VDR, which when activated by vitamin D binding decreases PTH gene transcription; on the contrary, decreasing levels of vitamin D or vitamin D deficiency stimulate PTH synthesis. ${ }^{8}$ VDRs are expressed in several tissues, such as the intestines (where calcium and phosphate absorption is regulated), the kidneys (where calcium reabsorption is regulated), and in bone (where calcium mobilization is regulated). ${ }^{9}$ VDR activation by the active form of vitamin D, calcitriol, also elevates parathyroid expression of the CaSR. ${ }^{4-7}$ Moreover, VDR activation in the gastrointestinal tract increases calcium absorption, thus elevating serum calcium levels and controlling parathyroid gland activity through CaSR activation. ${ }^{8,10}$ Serum phosphorus and phosphorus intake also affect calcitriol levels, primarily through modulation of the activity of 25-hydroxyvitamin $\mathrm{D}_{3}-1 \alpha$-hydroxylase and PTH expression by the parathyroid. Phosphate also stimulates production of the phosphatonin and fibroblast growth factor 23 (FGF-23) in osteocytes and osteoblasts. ${ }^{11}$ FGF-23 binds to a receptor complex consisting of FGFR1 and the transmembrane protein Klotho and modulates phosphate reabsorption, PTH synthesis, and vitamin D production. In turn, both PTH and FGF-23 regulate phosphate reabsorption by affecting the local density of the type 2 sodium-dependent phosphate cotransporter in the renal proximal tubules. ${ }^{11,12}$

Hyperphosphatemia, hypocalcemia, and low calcitriol levels that occur as kidney function declines are major players in the pathogenesis of SHPT in patients with CKD. ${ }^{11,13}$ Low calcium levels can downregulate the cellular expression of CaSR after the parathyroid cell proliferation. Compensatory downregulation of the CaSR and the VDR in the parathyroid may play a central role in the development of mineral disturbances in SHPT. ${ }^{11,13}$ Hyperphosphatemia

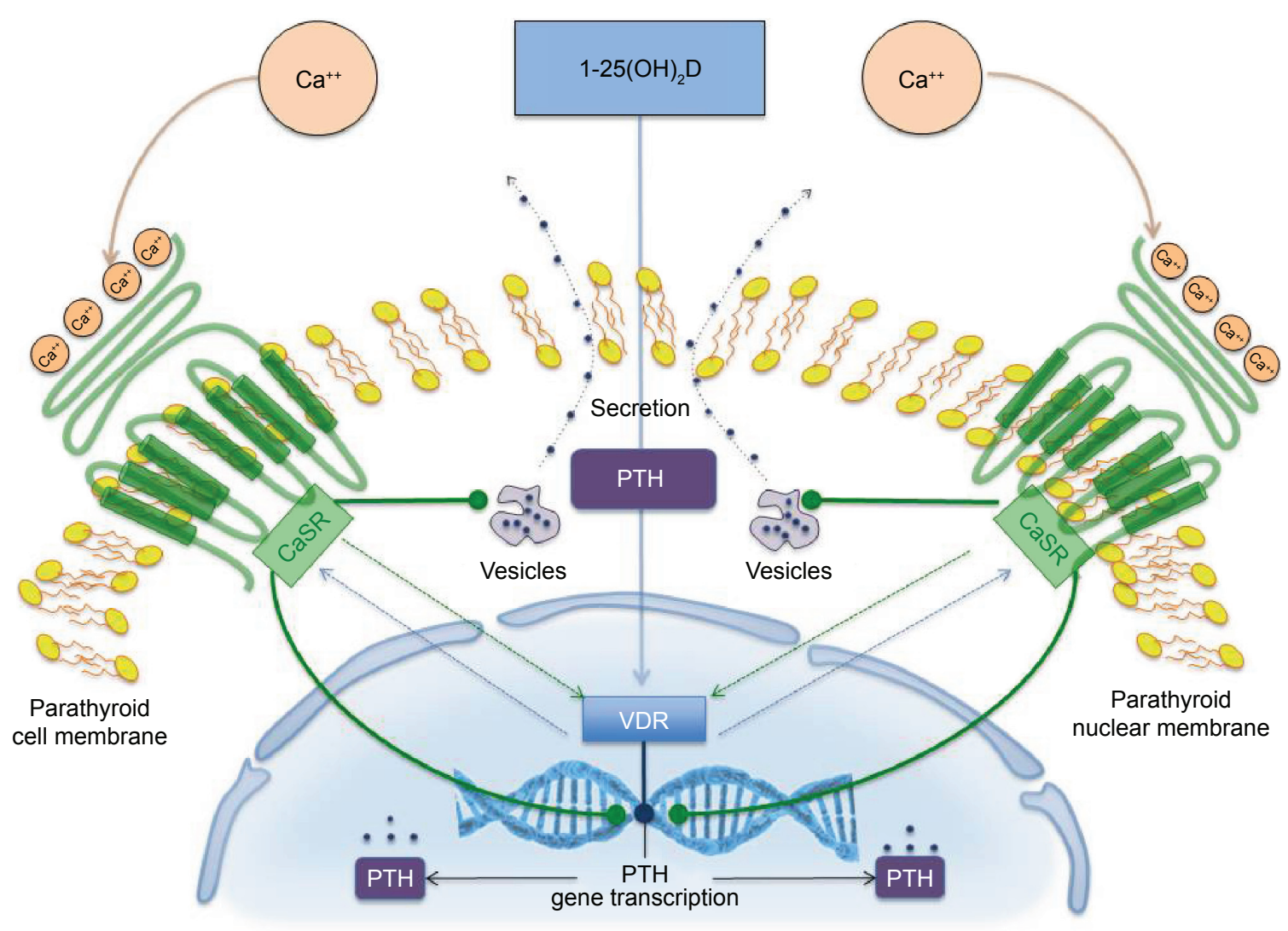

Figure I Regulation of PTH synthesis and secretion by CaSR and VDR in parathyroid glands.

Abbreviations: $\mathrm{Ca}^{++}$, ionized calcium; CaSR, calcium-sensing receptor; PTH, parathyroid hormone; VDR, vitamin D receptor. 
directly causes increased PTH synthesis and secretion, and parathyroid hyperplasia, with downregulation of both CaSR and VDR expression in SHPT. ${ }^{14}$ Additionally, high serum phosphate levels inhibit the activity of 25-hydroxyvitamin $\mathrm{D}_{3}-1 \alpha$-hydroxylase, the enzyme that converts vitamin $\mathrm{D}$ to its active form, calcitriol, thus disrupting the feedback loop acting through the CaSR and VDR that suppresses PTH., ${ }^{5,11}$ Persistent alterations in the signaling of the CaSR and VDR, including the reduced expression of both, can eventually result in refractory SHPT, in which the parathyroid gland becomes insensitive to calcium- or calcitriol-mediated inhibition of PTH synthesis. ${ }^{4,10}$ As kidney function declines in CKD, levels of FGF-23 are also increased. ${ }^{11}$ This has been attributed, at least in part, to reduced levels of Klotho in the kidney and parathyroid gland that occur as kidney function declines. ${ }^{15,16}$ Two additional factors that have been associated with disease progression and cardiovascular disease in CKD are indoxyl sulfate and p-cresyl sulfate, products of colonic bacterial fermentation of dietary proteins. ${ }^{17}$

Continuous stimulation of the parathyroid glands by these derangements in mineral homeostasis promotes PTH synthesis and eventually parathyroid hyperplasia. ${ }^{11}$ Because of their integral roles in the pathogenesis of SHPT, the CaSR, the VDR, and FGF-23 are biologically plausible targets for emerging therapies in the treatment of this disorder.

\section{Current treatment options}

SHPT is a common, serious, and costly manifestation of CKD, with negative effects on patient outcomes, especially on HD. With current treatment approaches, a considerable proportion of patients have inadequately controlled PTH, phosphorus, and/or calcium levels, and the ranges of these often fall outside of recommended ranges. ${ }^{18}$ Data from the Dialysis Outcomes and Practice Patterns Study (DOPPS) of patients receiving hemodialysis for $>180$ days support a greater risk of cardiovascular and all-cause mortality in patients with calcium levels $>10 \mathrm{mg} / \mathrm{dL}$, phosphorus levels $>7 \mathrm{mg} / \mathrm{dL}$, and PTH levels $>600 \mathrm{pg} / \mathrm{mL}$; risks are likewise increased in patients with combinations of these high-risk categories. ${ }^{19,20}$

Current treatment for SHPT should follow three steps: reduction of phosphorus uptake by dietary restriction or the use of phosphate binders; control of PTH with the use of vitamin D metabolites; and the use of calcimimetics, currently agents that allosterically modify the CaSR to enhance activation in the presence of circulating levels of calcium, thus reducing PTH (Table 1). ${ }^{21}$ Parathyroidectomy is usually a treatment strategy of last resort, after pharmacotherapy has failed. ${ }^{11}$ The goal of treatment is to maintain serum calcium, serum phosphorus, and PTH within accepted targeted ranges. ${ }^{22}$ Because of the limitations associated with the standard-of-care treatment for SHPT, PTH targets are not met for many patients. $^{23}$

\section{Low-phosphorus diet and phosphate binders}

Current guidelines suggest maintaining serum calcium and phosphorous with the normal range via balanced diet and/or phosphate binders administration. ${ }^{22}$ The use of vegetarian products as well as protein restriction is commonly suggested to limit phosphate intake. ${ }^{22}$ Cooking methods such as boiling are also proposed to limit $(20 \%-70 \%)$ the amount of phosphate contained in different foods. ${ }^{23}$ A recent meta-analysis suggested a significant reduction of phosphate levels of about $0.8 \mathrm{mg} / \mathrm{dL}$ (95\% confidence interval [CI]: -1.43 to -0.18$){ }^{24}$

In spite of these data, phosphate binders have long been perceived as a cornerstone in the treatment of SHPT. ${ }^{1}$ Indeed, due to poor treatment adherence, diet is often insufficient to reach a desirable control of serum phosphate levels, and a wide range of phosphate binders are now available. ${ }^{25}$ Although effective, aluminum-based phosphate binders have been replaced by calcium and iron-based and polymer-based phosphate binders due to their toxicity. ${ }^{25}$ Current guidelines suggest to limit aluminum-based phosphate binders in case of severe hyperphosphatemia and for a short period of time. ${ }^{22}$ When compared to placebo, all available compounds have been shown to lower serum phosphate to similar extent. ${ }^{23}$ However, differences among drugs may exist. Calcium-free phosphate binders are associated with lower serum calcium, and differences in PTH control with various drugs have been reported. ${ }^{24}$ Preliminary data also suggest that phosphate restriction and calcium-free phosphate binders may reduce FGF-23. ${ }^{25}$ Although the clinical relevance of different biochemical profiles still needs to be elucidated, some lines of evidence suggest that calcium-based phosphate binders may accelerate vascular calcification deposition and progression when compared to calcium-free phosphate binders. ${ }^{26}$ Calcium supplements can represent a substantial portion of an individual's daily calcium intake and contribute to deposition of calcium crystals in soft tissues ${ }^{26}$ in individuals with normal renal function ${ }^{27}$ as well as renal function impairment. ${ }^{26}$ Also, evidence supports the notion that calcium-free phosphate binders are associated with a better survival when compared to calcium-based phosphate binders, ${ }^{28}$ although future efforts are still required to shed light on this vexing 


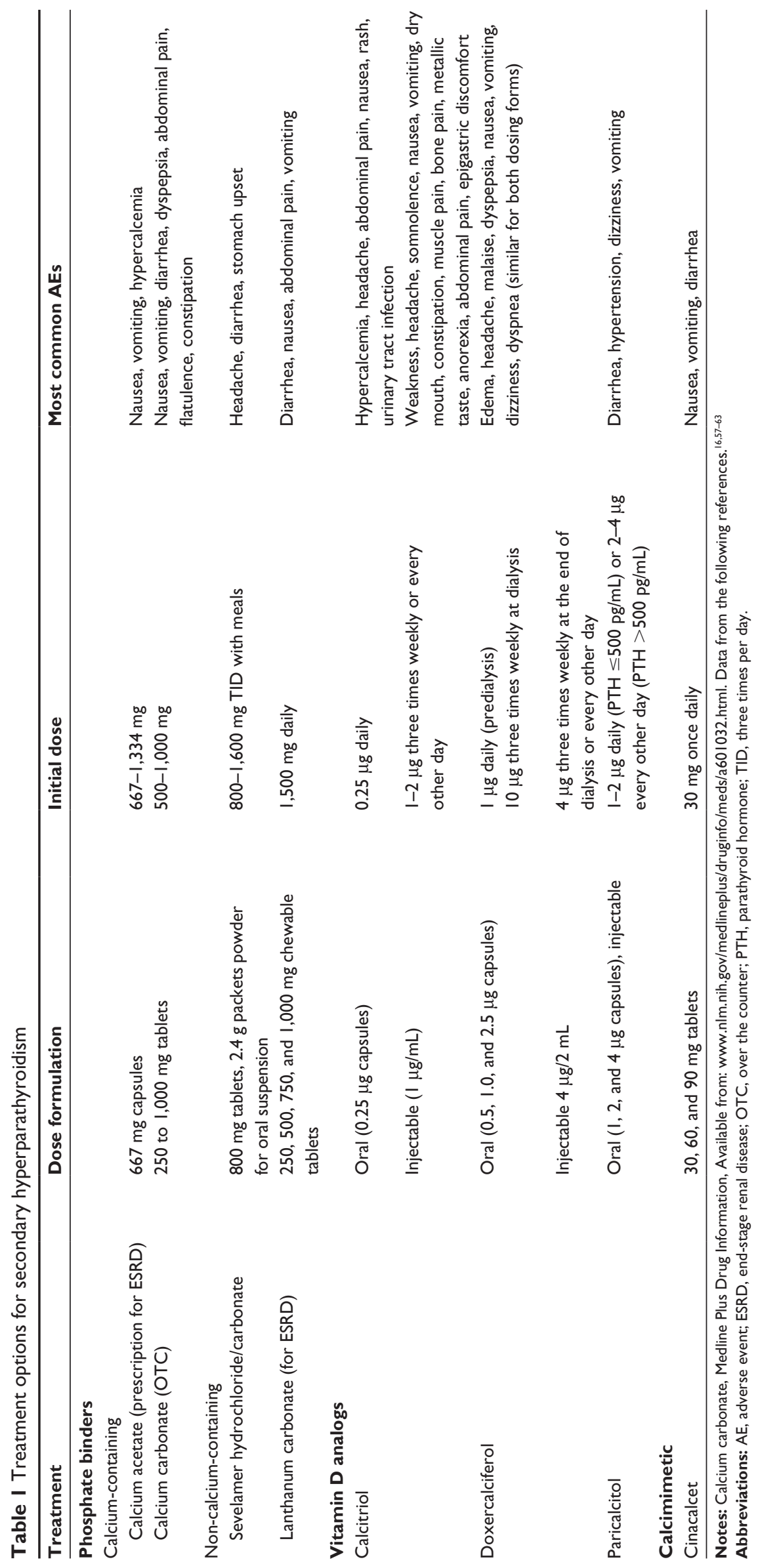


question. Similarly, future studies should investigate the impact of combination therapies for CKD-MBD on calcium and phosphate balance and their effect on outcome in CKD patients. ${ }^{29}$

\section{Calcimimetics}

Calcimimetics are a newer class of agents for the treatment of SHPT in patients receiving renal replacement therapy. ${ }^{30}$ This class of agents allosterically bind and activate the CaSR, increasing the response of the receptor to serum calcium (orthosteric agonist). ${ }^{22}$ To date, cinacalcet is the only commercially available drug of this class, although other molecules are under clinical development or about to hit the market. ${ }^{25,30}$ Although effective in PTH lowering, calcimimetics do not increase intestinal calcium or phosphate absorption. ${ }^{22,31}$ In the pivotal phase 3 clinical study, treatment of uncontrolled SHPT with cinacalcet or placebo for 26 weeks resulted in a greater proportion of patients in the calcimimetic arm achieving intact PTH levels $\leq 250 \mathrm{pg} / \mathrm{mL}$ (43\% versus $5 \%$ in the cinacalcet and placebo-treated study arms, respectively). ${ }^{30}$ Of interest, besides a greater overall PTH suppression, a better control of serum calcium and phosphorous was also noted. ${ }^{30}$ Additional studies further corroborate the PTH suppression efficacy of cinacalcet in combination with vitamin D or vitamin D analogs when compared with calcitriol as monotherapy. ${ }^{21}$ Sixty five $(65 \%)$ versus $36 \%$ of patients receiving cinacalcet or active vitamin D open-label monotherapy experienced a reduction in PTH from baseline of at least 30\% in the ACHIEVE study. ${ }^{32}$ In another open-label study of 16 weeks, $71 \%$ of patients receiving cinacalcet plus low-dose, active vitamin D treatment achieved PTH $\leq 300 \mathrm{pg} / \mathrm{mL}$ versus $22 \%$ of patients receiving vitamin $\mathrm{D}$ alone. ${ }^{33}$ Notably, in all these studies, mild-to-moderate hypocalcemia was a common, albeit easily managed, side effect attributed to CaSR modulation and likely a reduction in calcium mobilization from bone due to a tighter PTH control. ${ }^{11}$ Finally, the EVOLVE study, the largest placebo-controlled, double-blind clinical trial conducted in dialysis patients with SHPT also confirmed a greater achievement of biochemical parameters of CKDMBD among patients treated with cinacalcet compared to placebo. ${ }^{34,35}$ Although the primary end point of the study (ie, time until death, myocardial infarction, hospitalization for unstable angina, heart failure, or peripheral vascular event) was unmet, serum levels of intact PTH, calcium, phosphate, and FGF-23 were better controlled among patients allocated to cinacalcet. ${ }^{16,35}$ Notably, when compared to placebo, the most common complaints associated with treatment with calcimimetic were nausea, vomiting, and hypocalcemia ( $12 \%$ versus $2 \%$ of cinacalcet as opposed to placebo-treated patients experiencing hypocalcemia) ${ }^{16,34}$ that contributed to the slightly higher discontinuation rate due to adverse events (AEs) reported in the cinacalcet group (16\% versus $12 \%)$. CaSR modulation may exert beneficial effects beyond serum biochemical parameters control. ${ }^{36,37}$ Although inconclusive, the ADVANCE study (a phase 4 randomized clinical trial) showed a trend toward vascular calcification progression attenuation among subjects receiving low doses of active vitamin D and cinacalcet versus flexible doses of vitamin D to control SHPT. ${ }^{38}$ Overall, a small but not significant reduction in coronary artery calcification (CAC) progression was noted. However, patients adherent ${ }^{39}$ to the assigned therapy or with higher burden of CAC or cardiac valve calcification at study inception seem to benefit more from this combination theraphy. ${ }^{37,40}$ In a post hoc analysis, subjects with aortic valve calcification treated with CasR modulators experienced a 74\% risk reduction (adjusted odds ratio: 0.26; 95\% CI: 0.10-0.64) of CAC progression. ${ }^{40} \mathrm{As}$ in a previous study, the most common side effects reported by subjects assigned to cinacalcet treatment were gastrointestinal discomfort and hypocalcemia, affecting $21 \%$ and $7 \%$ of patients, respectively. ${ }^{37}$

Although CaSR modulation is an effective strategy to control SHPT, future efforts are needed to elucidate the effect interactions of different drugs to control CKD-MBD. In a recently published post hoc analysis ${ }^{38}$ of the INDEPENDENT study, a potentially deleterious effect of the combination of cinacalcet with calcium containing phosphate binders was shown. Indeed, among the 466 incident to dialysis patients recruited in the study, sevelamer but not calcium carbonate administration was associated with survival benefit. ${ }^{38}$ However, the concomitant administration of cinacalcet significantly modulated the impact of these phosphate binders on mortality (interaction test $P=0.006$ ). Coadministration of cinacalcet and calcium-free phosphate binders (hazard ratio: $0.34,95 \% \mathrm{CI}: 0.14-0.81, P=0.01$ for subjects treated with sevelamer and receiving cinacalcet or not) but not cinacalcet and calcium-containing phosphate binders (hazard ratio: $1.28,95 \% \mathrm{CI}$ : $0.82-2.00 ; P=0.26$ for subjects treated with calcium cinacalcet and receiving cinacalcet or not) was associated with improved survival. ${ }^{38}$ Although the hypothesis generating this piece of evidence suggests that calcium balance modulation may impact survival in $\mathrm{CKD}$, future efforts are needed to explore the effects of different combination therapies.

\section{Vitamin D and vitamin D derivatives}

In patients with CKD stages 3-5 (Table 2), both nutritional (cholecalciferol, ergocalciferol, calcifediol) and 
Table 2 Stages of chronic kidney disease

\begin{tabular}{lll}
\hline Stage & Definition & GFR $\left(\mathrm{mL} / \mathbf{m i n} / \mathbf{I . 7 3} \mathbf{~ m}^{2}\right)$ \\
\hline I & $\begin{array}{l}\text { Kidney damage and normal } \\
\text { or elevated GFR }\end{array}$ & $\geq 90$ \\
2 & $\begin{array}{l}\text { Kidney damage and mild } \\
\text { reduction in GFR }\end{array}$ & $60-89$ \\
3 & $\begin{array}{l}\text { Moderate reduction in GFR } \\
4\end{array}$ & $30-59$ \\
5 & Severe reduction in GFR & $15-29$ \\
\hline
\end{tabular}

Note: Data from Block et al. ${ }^{30}$

Abbreviation: GFR, glomerular filtration rate.

VDR activators (VDRAs) such as calcitriol and its analogs can improve abnormal mineral homeostasis. ${ }^{22}$ Although the optimal target of $25(\mathrm{OH}) \mathrm{D}$ remains uncertain, serum levels $>30 \mathrm{ng} / \mathrm{mL}$ are accepted as a normal threshold. ${ }^{41}$ Clinical data support a mild effect of nutritional vitamin D against nonsevere SHPT in CKD stages 3-5. ${ }^{42}$ International guidelines suggest the correction of nutritional vitamin D deficiency as a first-line therapy to counteract the onset and progression of CKD-MBD in predialysis patients. ${ }^{41,43,44}$ However, due to the inconsistency of current evidence on the best approach to correct 25(OH)D deficiency, the KDIGO, NICE, and ERBP guidelines are unable to provide any therapeutic suggestion on how to replenish low 25(OH) D levels. ${ }^{22,41,43}$ Doses originally recommended by KDOQI guidelines were often insufficient to achieve the expected 25(OH)D levels. ${ }^{45}$ Higher doses of cholecalciferol and ergocalciferol were thus suggested in $2012,{ }^{46}$ showing the superiority of cholecalciferol compared to ergocalciferol in replenishing 25(OH)D deficiency. ${ }^{47}$ Wetmore et al ${ }^{48}$ have recently randomized 44 nondialysis $\mathrm{CKD}$ patients to receive cholecalciferol 50,000 IU/wk versus ergocalciferol 50,000 IU/wk for 12 weeks, without suspending concomitant active therapy. Although cholecalciferol was more effective than ergocalciferol at raising serum $25(\mathrm{OH}) \mathrm{D}$ levels $(45 \pm 16.5$ $\mathrm{ng} / \mathrm{mL}$ and 30.7 versus $15.3 \mathrm{ng} / \mathrm{mL}, P<0.01$ ), changes in serum PTH or active vitamin D were similar between the groups, and a similar decline of $25(\mathrm{OH}) \mathrm{D}$ levels between the groups was observed after cessation of therapy ${ }^{48}$ Recent data support an innovative efficacy of a modified-release formulation of calcifediol, ${ }^{49}$ probably favored by its capability to raise 25(OH)D levels slowly enough for limiting an abrupt activation of vitamin D catabolism. ${ }^{50}$ This novel compound was effective in reducing iPTH levels in 44 predialysis CKD patients in a dose-dependent manner compared to placebo. ${ }^{49}$ Independently from the type and dose of nutritional vitamin D adopted, 25(OH)D levels should not exceed $100 \mathrm{ng} / \mathrm{mL}$, and particular caution should be paid while supplementing patients at high risk of 1- $\alpha$-hydroxylase activity, as kidney transplant patients and those affected by sarcoidosis or B-cell lymphoma. ${ }^{42}$ VDRA was shown to be effective in counteracting SHPT in nondialysis CKD patients. ${ }^{51}$ Selective VDRAs such as paricalcitol and doxercalciferol were developed to provide VDR activation with a lower risk of positive $\mathrm{Ca}$ and P balance. ${ }^{52}$ In a 12 -week, placebo-controlled, randomized study in patients receiving dialysis, paricalcitol effectively reduced PTH levels, with $6.7 \%$ of assessments indicative of hypercalcemia (serum calcium $>10.5 \mathrm{mg} / \mathrm{dL}$ ) versus $3.3 \%$ with placebo. ${ }^{53}$ Doxercalciferol has also been shown to be effective in reducing PTH in hemodialysis patients, with a modest but clinically notable incidence of hypercalcemia in $15 \%$ of assessments during treatment, ${ }^{54}$ while more recently a minimal increase in $\mathrm{Ca}$ and $\mathrm{P}$ levels was reported among patients receiving paricalcitol ${ }^{55}$ VDRAs are largely used to treat SHPT in dialysis patients. Improved survival among ESRD patients receiving VDRA was inconsistently reported in observational studies. ${ }^{56-58}$ Similarly, observational data showed heterogeneous results about the superiority of paricalcitol against nonselective VDRA in improving survival. ${ }^{59}$ Although selective VDRAs have been purported to limit calcitriol administration in CKD patients at higher cardiovascular risk, ${ }^{60}$ it must be noted that the impact of different VDRA on hard end points has never been tested in head-to-head RCTs. Due to the widespread genomic effect elicited by VDR activation, vitamin D is receiving growing interest for other targets than SHPT such as proteinuria, ${ }^{61,62}$ left ventricular hypertrophy (LVH), ${ }^{16,63}$ and anemia. ${ }^{64,65}$ Thus, vitamin D represents a culprit of medical therapy for CKDMBD. However, further studies are advocated to shed light on the many unresolved issues of this multifaceted therapy. ${ }^{66}$

\section{Parathyroidectomy}

Advances in the pharmacological treatment of SHPT often obviate parathyroidectomy; however, some researchers have reported that parathyroidectomy may be more cost-effective than cinacalcet in some patients with ESRD and uncontrolled SHPT. ${ }^{67}$ Successful parathyroidectomy can yield a dramatic reduction in PTH level and clinical symptoms. ${ }^{68}$ Dialysis patients undergoing parathyroidectomy have an increased risk of cardiopulmonary complications and mortality compared to patients not on dialysis. The risks of parathyroidectomy in dialysis patients are likely similar to other commonly performed procedures for dialysis patients. However, a retrospective review of dialysis patients with severe and unresponsive SHPT indicated that parathyroidectomy did not improve cardiovascular outcomes compared with standard medical treatment. ${ }^{69}$ In some instances, SHPT may continue 
after parathyroidectomy because of incomplete resection or because of ongoing PTH secretion from autotransplanted parathyroid tissue. ${ }^{11}$

\section{Current research goals}

As of November 30, 2016, 207 studies evaluating sSHPT were reported on ClinicalTrials.gov; 137, 26, and 34 studies are completed, open, or active but not recruiting patients, respectively. Of these 32 are observational and 175 interventional ( 14 on phase 1,32 on phase 2,40 on phase 3 , and 55 on phase $4 ; 34$ not defined). ${ }^{70,71}$ The largest proportion of these trials were for agents that modulate the activity of the CaSR (65 trials related to cinacalcet and 15 trials related to the new peptide CaSR agonist etelcalcetide [AMG 416]) or activate the VDR (paricalcitol, 94 trials). These findings reflect current interest in therapies that target pathways known to be involved in the development and progression of SHPT74. Etelcalcetide (AMG 416) is a new long-acting peptide calcimimetic agent composed of a linear chain of seven amino acids. ${ }^{72}$ It binds directly to CaSRs, inhibiting the production and the secretion of PTH by the parathyroid. ${ }^{73}$ This activity is due to the formation of a disulfide bridge between D-cysteine of etelcalcetide molecule and L-cysteine of the CaSRs, resulting in a rapid activation of the receptors. ${ }^{74}$

Several papers have been published in the last 2 years about pharmacological properties of etelcalcetide focusing on pharmacokinetics, biotransformation, and excretion of the drug in animal models ${ }^{2}$ and in CKD patients on hemodialysis treatment. ${ }^{75-77}$

In rats model with intact kidneys after intravenous dose, the drug is rapidly distributed to the tissues, and the renal elimination is the major removal pathway, while in binephrectomized rats the nonrenal elimination is only $1.2 \%{ }^{74}$ Etelcalcetide in whole blood is biotransformed by disulfide exchange to mainly form a covalent serum albumin peptide conjugate (SAPC). The biotransformation process in the human blood is reversible, but the rate of SAPC formation from etelcalcetide is faster than the inverse process. The preformation of SAPC also decreases the clearance rate during hemodialysis. ${ }^{75}$ When etelcalcetide was administered intravenously to CKD patients on hemodialysis, the treatment itself was the predominant clearance and elimination route, with a $60 \%$ of the administered dose recovered in the dialysate. ${ }^{72,75} \mathrm{As}$ a result of these observations, it was stated that etelcalcetide should be administered after the hemodialysis session to avoid elimination of a substantial fraction of the delivered dose. ${ }^{78}$ The efficacy of etelcalcetide was tested in several studies comparing the new molecule with placebo or cinacalcet in CKD patients suffering from SHPT on dialysis. In 340 patients receiving etelcalcetide versus 343 treated with oral cinacalcet, etelcalcetide reduced PTH value 50\% more than cinacalcet. ${ }^{9,79,80} \mathrm{In}$ another study, etelcalcetide administered thrice a week for as long as 26 weeks in 509 patients with moderate-to-severe SHPT, compared to 514 treated with placebo, resulted in a significantly greater reduction in PTH and FGF-23 values ( $70 \%$ versus $30 \%$ in placebo group), as well as an improvement in other markers of mineral metabolism. ${ }^{81,82}$ In a randomized double-blind placebo-controlled trial, patients, dived into three cohorts, received etelcalcetide $5 \mathrm{mg}$ or placebo for 2 weeks (cohort 1 ), or etelcalcetide $5 \mathrm{mg}$ or $10 \mathrm{mg}$ or placebo for 4 weeks (cohorts 2 and 3). The mean percentage change from baseline PTH levels was $-49 \%$ with etelcalcetide versus $+29 \%$ with placebo in cohort 2 (etelcalcetide $10 \mathrm{mg}$ ), while in cohort 3 (etelcalcetide $5 \mathrm{mg}$ ) the percentage change was $-33 \%$ versus $+2 \%$. Percentage of patients reaching a PTH value reduction of $\geq 30 \%$ was $76 \%$ in etelcalcetide $10 \mathrm{mg}$ cohort versus $10 \%$ in placebo $(P<0.0001)$. In the cohort with etelcalcetide $5 \mathrm{mg}$, the percentage was smaller (54\% versus $15 \%)$. The number of patients achieving at the end of the study protocol a PTH value of $\leq 300 \mathrm{pg} / \mathrm{mL}$ were $67 \%$ and $46 \%$ in etelcalcetide arm 10 and $5 \mathrm{mg}$, respectively. ${ }^{77,83}$ According to findings from this trial, there was a dose-dependent reduction in PTH values and a corresponding effect on the bone formation (bone-specific alkaline phosphatase) and bone resorption (tartrate-resistant acid phosphatase $5 \mathrm{~b}$ ) markers. ${ }^{84}$ The safety profile of etelcalcetide has been studied relative to a specific patient population (CKD patients on hemodialysis therapy) for whom the drug is intended. During phase 2 and phase 3 clinical studies, a total of 1,655 subjects received at least 1 dose of etelcalcetide, and 499 of them received etelcalcetide for over 1 year. The most common AE within the placebocontrolled studies was asymptomatic blood calcium reduction (63.8\% etelcalcetide; $10.1 \%$ placebo) ${ }^{76,85,86} \mathrm{~A}$ similar pattern was observed in the active-controlled study and consistent with the placebo-controlled studies. For the most frequently reported AEs, asymptomatic blood calcium reduction $(68.9 \%$ etelcalcetide; $59.8 \%$ cinacalcet) was the most important adverse effect, while gastrointestinal symptoms were prevalent in cinacalcet-treated patients compared to etelcalcetide (Table 1). Based on the Kaplan-Meier estimate, the median of the first occurrence of hypocalcemic event was 9.6 weeks in the placebo-controlled study and 9.6 weeks in active controlled study in the arm of etelcalcetide..$^{87,88}$

With regard to the incidence of serious AEs, these were less frequent $(21.4 \%)$ in patients with a PTH value of $>1,000 \mathrm{pg} / \mathrm{mL}$ at the screening compared with 
Table 3 Incidence (\%) of most frequent adverse drug reactions

\begin{tabular}{|c|c|c|c|c|}
\hline \multirow{2}{*}{$\begin{array}{l}\text { Most frequent } \\
\text { adverse events }\end{array}$} & \multicolumn{2}{|c|}{ Placebo-controlled studies } & \multicolumn{2}{|c|}{ Cinacalcet-controlled studies } \\
\hline & Placebo $n=5 \mid 3$ & Etelcalcetide $n=503$ & Cinacalcet $n=34$ I & Etelcalcetide $n=338$ \\
\hline Diarrhea & 8.6 & 10.7 & 10.3 & 6.2 \\
\hline Nausea & 6.2 & 10.7 & 22.6 & 18.3 \\
\hline Vomiting & 5.1 & 8.9 & 13.8 & 13.3 \\
\hline Calcium reduction & 10.1 & 63.8 & 59.8 & 68.9 \\
\hline Hypocalcemia $^{a}$ & 0.2 & 7.0 & 2.3 & 5.0 \\
\hline Hyperkalemia & 3.1 & 4.4 & 5.3 & 3.8 \\
\hline Muscle spasms & 6.6 & 11.5 & 5.9 & 6.5 \\
\hline Paraesthesia & 1.2 & 6.2 & 2.6 & 3.3 \\
\hline Hypotension & - & - & 2.9 & 6.8 \\
\hline
\end{tabular}

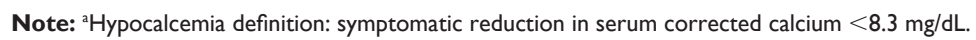

subjects with PTH $>600 \leq 1,000 \mathrm{pg} / \mathrm{mL}(26.2 \%)$ or $<600$ $\mathrm{pg} / \mathrm{mL}(28.2 \%)$. A similar behavior has been observed with respect to age, with a lower incidence of events related to serum calcium reduction $(56.5 \%)$ in subjects aged $\geq 65$ years compared to $<65$ years $(67.8 \%){ }^{88}$ In the pooled placebocontrolled trials, a QT prolongation $(500 \mathrm{~ms})$ secondary to hypocalcemia was observed in $4.8 \%$ of etelcalcetide and $1.9 \%$ of placebo subjects. For this reason, close monitoring of calcium levels is necessary in patients with a previous risk factor such as congenital long QT syndrome, previous history of QT prolongation, family history of long QT syndrome, or sudden cardiac death and other conditions that predispose to QT prolongation. ${ }^{88}$

Considering the overall safety profile of using etelcalcetide, we can see that the reported AEs are related to the mechanism of action of calcimimetic drugs and are somewhat similar to cinacalcet. The most important risk for etelcalcetide is hypocalcemia and other events that can occur secondary to reductions in serum calcium (ie, QTc prolongation, ventricular arrhythmia, convulsions, and worsening heart failure). These adverse effects, specially hypocalcemia and reduced calcium levels, occurred slightly more than with cinacalcet, possibly due to a more potent effect of etelcalcetide. However in dialysis patients, attending a dialysis unit thrice a week, regular monitoring of these parameters will be enough to prevent serious AEs. Despite bypassing the gastrointestinal tract, owing to intravenous administration, etelcalcetide only partially reduced gastrointestinal adverse effects (nausea and diarrhea) (Table 3) seen with cinacalcet use, supporting evidence that these effects are possibly related to a systemic action rather than a local one, mediated by CaSRs activation. ${ }^{89}$ Etelcalcetide intravenous administration at the end of the dialysis session, on the contrary, would ameliorate substantially the adherence to the SHPT treatment by the patients, also reducing the burden of pills of drug therapy.
On the other hand, the superiority of etelcalcetide versus cinacalcet in reducing the levels of sPTH can offer a viable chance to demonstrate the role of PTH on the cardiovascular risk of patients on dialysis. ${ }^{89,90}$ The possibility of normalizing, with the new available drugs, mineral metabolism in uremic patients on dialysis may reduce bias related to the problem of therapeutic compliance in RCTs and help to clarify the effect of biochemical normalization on patients' survival, even if it will be necessary to clearly identify the patients to whom this therapy should be offered, and this is also related to the economic capacity of health systems in the various countries. ${ }^{91,92}$ The EMA Assessment Report (Procedure No EMEA/H/C/003995/0000) related to "Parsabiv" (Amgen Inc, Thousand Oaks, CA, USA) (etelcalcetide), after careful benefit-risk assessment, argues that overall, the benefits of etelcalcetide in the treatment of secondary HPT in CKD patients are considered to outweigh the risks, recommending accurate controls of safety in the postmarketing period. ${ }^{87}$

\section{Scientific rationale}

Vitamin D analogs, such as paricalcitol, selectively target the VDR, lowering PTH levels. ${ }^{2}$ However, clinical data reveal that paricalcitol treatment can be associated with elevation in serum calcium and phosphorus, and a significant minority of patients treated with paricalcitol experience hypercalcemia. ${ }^{16}$ Calcimimetics, such as cinacalcet, lower PTH levels and reduce serum calcium and phosphorus. ${ }^{15,21,22,30}$ However, cinacalcet is administered orally and has been associated with gastrointestinal AEs in approximately $30 \%$ of patients and with hypocalcemia in $7 \% .{ }^{21}$ In addition, poor adherence has been observed among dialysis patients self-administering oral cinacalcet. ${ }^{93}$ Consequently, there has been interest in the development of novel CaSR agonists with improved tolerability and vitamin D derivatives with reduced calcemic actions to further advance the treatment of SHPT. 


\section{Conclusion}

The pharmacological management of SHPT has progressed in recent years. The introduction of targeted therapies, such as selective VDR and CaSR modulators, offers an increased opportunity to adequately control elevated PTH, especially in patients with CKD receiving dialysis. Emerging therapies for SHPT, such as etelcalcetide (AMG 416) administered intravenously, may promote patient adherence, simplify treatment, and, in turn, improve the likelihood of achieving treatment goals. However, an open question remains as to whether emerging treatments for SHPT will reduce the risk of clinical end points such as cardiovascular morbidity and mortality. If emerging therapies for SHPT can reduce PTH, restore mineral homeostasis, promote adherence, and reduce the risk of troublesome side effects, they may provide the prerequisite features for improving long-term outcomes in patients with SHPT receiving dialysis.

\section{Disclosure}

Antonio Bellasi has received speaking honoraria or consultancy fees from Amgen, Sanofi, and Sanifit. The authors report no other conflicts of interest in this work.

\section{References}

1. Saliba W, El-Haddad B. Secondary hyperparathyroidism: pathophysiology and treatment. J Am Board Fam Med. 2009;22:574-581.

2. Brown AJ, Slatopolsky E. Drug insight: vitamin D analogs in the treatment of secondary hyperparathyroidism in patients with chronic kidney disease. Nat Clin Pract Endocrinol Metab. 2007;3:134-144.

3. Joy MS, Karagiannis PC, Peyerl FW. Outcomes of secondary hyperparathyroidism in chronic kidney disease and the direct costs of treatment J Manag Care Pharm. 2007;13:397-411.

4. McCann LM, Beto J. Roles of calcium-sensing receptor and vitamin D receptor in the pathophysiology of secondary hyperparathyroidism. J Ren Nutr. 2010;20:141-150.

5. Moe SM, Drueke TB. Management of secondary hyperparathyroidism: the importance and the challenge of controlling parathyroid hormone levels without elevating calcium, phosphorus, and calcium-phosphorus product. Am J Nephrol. 2003;23:369-379.

6. Cozzolino M, Urena-Torres P, Vervloet MG, et al. Is chronic kidney disease-mineral bone disorder (CKD-MBD) really a syndrome? Nephrol Dial Transplant. 2014;29(10):1815-1820.

7. Riccardi D, Brown EM. Physiology and pathophysiology of the calcium-sensing receptor in the kidney. Am J Physiol Renal Physiol. 2010;298:F485-F499.

8. Gallieni M, Cozzolino M, Fallabrino G, et al. Vitamin D: physiology and pathophysiology. Int J Artif Organs. 2009;32:87-94.

9. Evenepoel P, Rodriguez M, Ketteler M. Laboratory abnormalities in CKD-MBD: markers, predictors, or mediators of disease? Semin Nephrol. 2014;34:151-163.

10. Wetmore JB, Quarles LD. Treatment of secondary hyperparathyroidism in kidney disease: what we know and do not know about use of calcimimetics and vitamin D analogs. Int J Nephrol Renovasc Dis. 2008;1:5-17.

11. Cunningham J, Locatelli F, Rodriguez M. Secondary hyperparathyroidism: pathogenesis, disease progression, and therapeutic options. Clin J Am Soc Nephrol. 2011;6:913-921.

12. Goodman WG, Quarles LD. Development and progression of secondary hyperparathyroidism in chronic kidney disease: lessons from molecular genetics. Kidney Int. 2008;74:276-288.
13. Cozzolino M, Ciceri P, Volpi EM, et al. Pathophysiology of calcium and phosphate metabolism impairment in chronic kidney disease. Blood Purif. 2009;27:338-344.

14. Nakajima K, Umino K, Azuma Y, et al. Stimulating parathyroid cell proliferation and PTH release with phosphate in organ cultures obtained from patients with primary and secondary hyperparathyroidism for a prolonged period. J Bone Miner Metab. 2009;27:224-233.

15. Drueke TB, Massy ZA. Circulating Klotho levels: clinical relevance and relationship with tissue Klotho expression. Kidney Int. 2013;83:13-15.

16. Moe SM, Thadhani R. What have we learned about chronic kidney disease-mineral bone disorder from the EVOLVE and PRIMO trials? Curr Opin Nephrol Hypertens. 2013;22:651-655.

17. Rossi M, Campbell KL, Johnson DW. Indoxyl sulphate and p-cresyl sulphate: therapeutically modifiable nephrovascular toxins. $O A \mathrm{Neph}$ rology. 2013;1:13.

18. Young EW, Albert JM, Satayathum S, et al. Predictors and consequences of altered mineral metabolism: the Dialysis Outcomes and Practice Patterns Study. Kidney Int. 2005;67:1179-1187.

19. Tentori F, Blayney MJ, Albert JM, et al. Mortality risk for dialysis patients with different levels of serum calcium, phosphorus, and PTH: the Dialysis Outcomes and Practice Patterns Study (DOPPS). Am J Kidney Dis. 2008;52:519-530.

20. Fukagawa M, Kido R, Komaba H, et al. Abnormal mineral metabolism and mortality in hemodialysis patients with secondary hyperparathyroidism: evidence from marginal structural models used to adjust for time-dependent confounding. Am J Kidney Dis. 2014;63:979-987.

21. Stubbs JR, Wetmore JB. Does it matter how parathyroid hormone levels are suppressed in secondary hyperparathyroidism? Semin Dial. 2011;24:298-306.

22. KDIGO clinical practice guideline for the diagnosis, evaluation, prevention, and treatment of Chronic Kidney Disease-Mineral and Bone Disorder (CKD-MBD). Kidney Int Suppl. 2009;(113):S1-S130.

23. Galassi A, Cupisti A, Santoro A, Cozzolino M. Phosphate balance in ESRD: diet, dialysis and binders against the low evident masked pool. J Nephrol. 2015;28:415-429.

24. Sekercioglu N, Angeliki Veroniki A, Thabane L, et al. Effects of different phosphate lowering strategies in patients with CKD on laboratory outcomes: a systematic review and NMA. PLoS One. 2017;12(3): e0171028

25. Cozzolino M, Tomlinson J, Walsh L, Bellasi A. Emerging drugs for secondary hyperparathyroidism. Expert Opin Emerg Drugs. 2015;20(2): 197-208.

26. Cozzolino M, Mazzaferro S, Brandenburg V. The treatment of hyperphosphataemia in CKD: calcium-based or calcium-free phosphate binders? Nephrol Dial Transplant. 2011;26:402-407.

27. Anderson JJ, Kruszka B, Delaney JA, et al. Calcium intake from diet and supplements and the risk of coronary artery calcification and its progression among older adults: 10-year follow-up of the Multi-Ethnic Study of Atherosclerosis (MESA). J Am Heart Assoc. 2016;5:e003815.

28. Palmer SC, Gardner S, Tonelli M, et al. Phosphate-binding agents in adults with CKD: a network meta-analysis of randomized trials. $\mathrm{Am} \mathrm{J}$ Kidney Dis. 2016;68(5):691-702.

29. di Filippo S, Carfagna F, la Milia V, et al. Assessment of intradialysis calcium mass balance by single pool variable-volume calcium kinetic model. Hemodial Int. Epub 2017 Feb 5.

30. Block GA, Martin KJ, de Francisco AL, et al. Cinacalcet for secondary hyperparathyroidism in patients receiving hemodialysis. $N$ Engl J Med. 2004;350:1516-1525.

31. Bellasi A, Cozzolino M. Cinacalcet: the chemical parathyroidectomy? Clin Kidney J. 2013;6(3):253-256.

32. Fishbane S, Shapiro WB, Corry DB, et al. Cinacalcet $\mathrm{HCl}$ and concurrent low-dose vitamin D improves treatment of secondary hyperparathyroidism in dialysis patients compared with vitamin D alone: the ACHIEVE study results. Clin J Am Soc Nephrol. 2008;3:1718-1725.

33. Messa P, Macario F, Yaqoob M, et al. The OPTIMA study: assessing a new cinacalcet (Sensipar/Mimpara) treatment algorithm for secondary hyperparathyroidism. Clin J Am Soc Nephrol. 2008;3:36-45. 
34. Evolve Trial Investigators. Effect of cinacalcet on cardiovascular disease in patients undergoing dialysis. $N$ Engl J Med. 2012;367:2482-2494.

35. Parfrey PS, Chertow GM, Block GA, et al. The clinical course of treated hyperparathyroidism among patients receiving hemodialysis and the effect of cinacalcet: the EVOLVE trial. J Clin Endocrinol Metab. 2013; 98:4834-4844.

36. Bellasi A, Cozzolino M, Adragao T, et al. Phosphate binders in moderate chronic kidney disease: where do we stand? J Nephrol. 2013;26: 993-1000.

37. Raggi P, Chertow GM, Torres PU, et al. The ADVANCE study: a randomized study to evaluate the effects of cinacalcet plus low-dose vitamin D on vascular calcification in patients on hemodialysis. Nephrol Dial Transplant. 2011;26:1327-1339.

38. Bellasi A, Reiner M, Pétavy F, Goodman W, Floege J, Raggi P. Presence of valvular calcification predicts the response to cinacalcet: data from the ADVANCE study. J Heart Valve Dis. 2013;22(3): 391-399.

39. Ureña-Torres PA, Floege J, Hawley CM, et al. Protocol adherence and the progression of cardiovascular calcification in the ADVANCE study. Nephrol Dial Transplant. 2013;28(1):146-152.

40. Bellasi A, Cozzolino M, Russo D, Molony D, Di Iorio B. Cinacalcet but not vitamin D use modulates the survival benefit associated with sevelamer in the INDEPENDENT study. Clin Nephrol. 2016;86(9): $113-124$.

41. Goldsmith DJ, Covic A, Fouque D, et al. Endorsement of the Kidney Disease Improving Global Outcomes (KDIGO) Chronic Kidney Disease-Mineral and Bone Disorder (CKD-MBD) Guidelines: a European Renal Best Practice (ERBP) commentary statement. Nephrol Dial Transplant. 2010;25(12):3823-3831.

42. Morrone LF, Bolasco P, Camerini C, et al. Vitamin D in patients with chronic kidney disease: a position statement of the Working Group "Trace Elements and Mineral Metabolism" of the Italian Society of Nephrology. J Nephrol. 2016;29:305-328.

43. NICE Chronic kidney disease in adults: assessment and management clinical guideline Pub. Available from: https://nice.org.uk/guidance/ cg182. Accessed July 23, 2014.

44. KDIGO 2012 Clinical practice guideline for the evaluation and management of chronic kidney disease. Kidney Int Suppl. 2013;3(1): $1-150$.

45. Kandula P, Dobre M, Schold JD, Schreiber MJ Jr, Mehrotra R, Navaneethan SD. Vitamin D supplementation in chronic kidney disease: a systematic review and meta-analysis of observational studies and randomized controlled trials. Clin J Am Soc Nephrol. 2011;6: $50-62$.

46. Nigwekar SU, Bhan I, Thadhani R. Ergocalciferol and cholecalciferol in CKD. Am J Kidney Dis. 2012;60(1):139-156.

47. Tripkovic L, Lambert H, Hart K, et al. Comparison of vitamin D2 and vitamin D3 supplementation in raising serum 25-hydroxyvitamin D status: a systematic review and meta-analysis. Am J Clin Nutr. 2012; 95(6):1357-1364.

48. Wetmore JB, Kimber C, Mahnken JD, Stubbs JR, Cholecalciferol V. Ergocalciferol for 25-hydroxyvitamin D (25(OH)D) repletion in chronic kidney disease: a randomised clinical trial. Br J Nutr. 2016; 116(12):2074-2081.

49. Sprague SM, Crawford PW, Melnick JZ, et al. Use of extended-release calcifediol to treat secondary hyperparathyroidism in stages 3 and 4 chronic kidney disease. Am J Nephrol. 2016;44(4):316-325.

50. Petkovich M, Melnick J, White J, Tabash S, Strugnell S, Bishop CW. Modified-release oral calcifediol corrects vitamin D insufficiency with minimal CYP24A1 upregulation. J Steroid Biochem Mol Biol. 2015;148: 283-289.

51. Hamdy NA, Kanis JA, Beneton MN, et al. Effect of alfacalcidol on natural course of renal bone disease in mild to moderate renal failure. BMJ. 1995;310(6976):358-363

52. Brown AJ. Vitamin D analogs for secondary hyperparathyroidism: what does the future hold? J Steroid Biochem Mol Biol. 2007;103(3-5): $578-583$.
53. Martin KJ, González EA, Gellens M, Hamm LL, Abboud H, Lindberg J. 19-Nor-1-alpha-25-dihydroxyvitamin D2 (Paricalcitol) safely and effectively reduces the levels of intact parathyroid hormone in patients on hemodialysis. J Am Soc Nephrol. 1998;9(8):1427-1432.

54. Frazao JM, Chesney RW, Coburn JW. Intermittent oral 1alphahydroxyvitamin D2 is effective and safe for the suppression of secondary hyperparathyroidism in haemodialysis patients. 1alphaD2 Study Group. Nephrol Dial Transplant. 1998;13(Suppl 3):68-72.

55. Coyne DW, Goldberg S, Faber M, Ghossein C, Sprague SM. A randomized multicenter trial of paricalcitol versus calcitriol for secondary hyperparathyroidism in stages 3-4 CKD. Clin J Am Soc Nephrol. 2014; 9(9):1620-1626.

56. Messa P, Cozzolino M, Brancaccio D, et al; FARO Study Group. Effect of VDRA on survival in incident hemodialysis patients: results of the FARO-2 observational study. BMC Nephrol. 2015;16:11.

57. Kovesdy CP, Kalantar-Zadeh K. Vitamin D receptor activation and survival in chronic kidney disease. Kidney Int. 2008;73(12):1355-1363.

58. Tentori F, Albert JM, Young EW, et al. The survival advantage for haemodialysis patients taking vitamin $\mathrm{D}$ is questioned: findings from the Dialysis Outcomes and Practice Patterns Study. Nephrol Dial Transplant. 2009;24(3):963-972.

59. Tentori F, Hunt WC, Stidley CA, et al; Medical Directors of Dialysis Clinic Inc. Mortality risk among hemodialysis patients receiving different vitamin D analogs. Kidney Int. 2006;70(10):1858-1865.

60. Mazzaferro S, Goldsmith D, Larsson TE, Massy ZA, Cozzolino M. Vitamin D metabolites and/or analogs: which D for which patient? Curr Vasc Pharmacol. 2014;12(2):339-349.

61. Kim MJ, Frankel AH, Donaldson M, et al. Oral cholecalciferol decreases albuminuria and urinary TGF- $\beta 1$ in patients with type 2 diabetic nephropathy on established renin-angiotensin-aldosterone system inhibition. Kidney Int. 2011;80(8):851-860.

62. de Borst MH, Hajhosseiny R, Tamez H, Wenger J, Thadhani R, Goldsmith DJ. Active vitamin D treatment for reduction of residual proteinuria: a systematic review. $J$ Am Soc Nephrol. 2013;24(11): $1863-1871$

63. Thadhani R, Appelbaum E, Pritchett Y, et al. Vitamin D therapy and cardiac structure and function in patients with chronic kidney disease: the PRIMO randomized controlled trial. JAMA. 2012;307(7):674-684.

64. Brancaccio D, Cozzolino M, Gallieni M. Hyperparathyroidism and anemia in uremic subjects: a combined therapeutic approach. J Am Soc Nephrol. 2004;15(Suppl 1):S21-S24.

65. Riccio E, Sabbatini M, Bruzzese D, et al. Effect of paricalcitol vs calcitriol on hemoglobin levels in chronic kidney disease patients: a randomized trial. PLoS One. 2015;10(3):e0118174

66. Galassi A, Bellasi A, Auricchio S, Papagni S, Cozzolino M. Which vitamin $\mathrm{D}$ in CKD-MBD? The time of burning questions. Biomed Res Int. 2013;2013:864012.

67. Narayan R, Perkins RM, Berbano EP, et al. Parathyroidectomy versus cinacalcet hydrochloride-based medical therapy in the management of hyperparathyroidism in ESRD: a cost utility analysis. Am J Kidney Dis. 2007;49:801-813

68. Conzo G, Perna AF, Savica V, et al. Impact of parathyroidectomy on cardiovascular outcomes and survival in chronic hemodialysis patients with secondary hyperparathyroidism. A retrospective study of 50 cases prior to the calcimimetics era. BMC Surg. 2013;13(Suppl 2):S4.

69. Di Iorio B, Molony D, Bell C, et al; INDEPENDENT Study Investigators. Sevelamer versus calcium carbonate in incident hemodialysis patients: results of an open-label 24-month randomized clinical trial. Am J Kidney Dis. 2013;62(4):771-778.

70. ClinicalTrials.gov [homepage on the Internet]. Secondary Hyperparathyroidism. Available from: https://clinicaltrials.gov. Accessed November 30, 2016.

71. Bover J, Ureña-Torres P, Lloret MJ, et al. Integral pharmacological management of bone mineral disorders in chronic kidney disease (part II): from treatment of phosphate imbalance to control of PTH and prevention of progression of cardiovascular calcification. Expert Opin Pharmacother. 2016;17(10):1363-1373. 
72. Blair HA. Etelcalcetide: first global approval. Drugs. 2016;76(18): 1787-1792.

73. Martin KJ, Bell G, Pickthorn K, et al. Velcalcetide (AMG 416), a novel peptide agonist of the calcium-sensing receptor, reduces serum parathyroid hormone and FGF23 levels in healthy male subjects. Nephrol Dial Transplant. 2014;29:385-392.

74. Subramanian R, Zhu X, Kerr SJ, et al. Nonclinical pharmacokinetics disposition, and drug-drug interaction potential of a novel d-amino acid peptide agonist of the calcium sensing receptor AMG 416 (Etelcalcetide). Drug Metab Dispos. 2016;44(8):1319-1331.

75. Subramanian R, Zhu X, Hock MB, et al. Pharmacokinetics, biotransformation, and excretion of [14C]Etelcalcetide (AMG 416) following a single microtracer intravenous dose in patients with chronic kidney disease on hemodialysis. Clin Pharmacokinetics. 2017; 56(2):179-192.

76. Martin KJ, Block GA, Cheng S, et al. Comparison of the Efficacy and Safety of Intravenous (IV) Etelcalcetide (AMG 416) and Oral Cinacalcet (CIN) in Patients on Hemodialysis (HD) with Secondary Hyperparathyroidism (sHPT). Abstract Poster presented at: Kidney Week; November 7; 2015; San Diego, CA.

77. Chen P, Olsson Gisleskog P, Perez-Ruixo JJ, et al. Population pharmacokinetics and pharmacodynamics of the calcimimetic Etelcalcetide in chronic kidney disease and secondary hyperparathyroidism receiving. CPT Pharmacometrics Syst Pharmacol. 2016;5:484-494.

78. Edson KZ, Wu BM, Iyer A, Goodman W, Skiles GL, Subramanian R. Determination of Etelcalcetide biotransformation and hemodialysis kinetics to guide the timing of its dosing. Kidney Int Rep. 2016;1: 24-33.

79. Block GA, Bushinsky DA, Cheng S, et al. Effect of Etelcalcetide vs cinacalcet on serum parathyroid hormone in patients receiving hemodialysis with secondary hyperparathyroidism a randomized clinical trial. JAMA. 2017;317(2):156-164.

80. Fukagawa M, Yokoyama K, Shigematsu T, et al; ONO-5163 Study Group Efficacy and Safety of a Novel Intravenous Calcimimetic. ONO5163 (ETELCALCETIDE) in Japanese hemodialysis patients with secondary hyperparathyroidism: results of a placebo controlled phase III study. Nephrol Dial Transplant. 2016;31(Suppl 1):i454-i471.

81. Cunningham J. A long acting intravenous calcimimetic (AMG416) for secondary hyperparathyroidism (SHPT) in haemodialysed patients. Poster presented at: 52 nd ERA-EDTA Congress; 2015; London, UK.

82. Stollenwerk B, Briggs A, Dehmel B, et al. Modelling Etelcalcetide effectiveness on health outcomes: relating biochemical outcomes to mortality, cardiovascular events, fractures and parathyroidectomy. Abstract Poster presented at: 16th Biennial European Conference SMDM; June 12-24; 2016; London, UK.
83. Bell G, Huang S, Oaks T, Martin KJ, Block GA. A randomized, doubleblind, phase 2 study evaluating the safety and efficacy of AMG 416 for the treatment of secondary hyperparathyroidism in hemodialysis patients. Curr Med Res Opin. 2015;31(5):943-952.

84. Yokoyama K, Fukagawa M, Shigematsu T, et al; ONO-5163 Study Group A novel Intravenous Calcimimetics. ONO-5163 (ETELCALCETIDE) a multicenter single and multiple dose study in Japanese hemodialysis patients with secondary hyperparathyroidism. Nephrol Dial Transplant. 2016;31(Suppl 1):i454-i471.

85. Block GA, Bushinsky DA, Cunningham J, et al. Effect of Etelcalcetide vs placebo on serum parathyroid hormone in patients receiving hemodialysis with secondary hyperparathyroidism two randomized clinical trials. JAMA. 2017;317(2):146-155.

86. Bushinsky DA, Block GA, Martin KJ, et al. Treatment of secondary hyperparathyroidism: results of a phase 2 trial evaluating an intravenous peptide agonist of the calcium-sensing receptor. Am J Nephrol. 2015;42(5):379-388.

87. Bushinsky DA, Block GA, Cheng S, et al. One year efficacy and safety of intravenous (IV) ETELCALCETIDE (AMG 416) in patients on hemodialysis (HD) with secondary hyperparathyroidism (SHPT). Abstract Poster presented at: 53rd ERA-EDTA Congress; May 21-24; 2016; Vienna, Austria.

88. European Medicines Agency [homepage on the Internet]. Parsabiv: summary of product characteristics. Available from: http://www.ema. europa.eu. Accessed November 30, 2016.

89. Middleton JP, Wolf M. Second chances to improve ESRD outcomes with a second-generation calcimimetic. JAMA. 2017;317(2):139-141.

90. Harada K. ETELCALCETIDE(ONO-5163, AMG 416), an intravenously available allosteric modulator of the calcium sensing receptor, effectively controls parathyroid hormone levels in a rat chronic renal insufficiency model with secondary hyperparathyroidism. Nephrol Dial Transplant. 2016;31(Suppl 1):i246-i258.

91. Stollenwerk B, Iannazzo S, Akehurst R, et al. Assessing the cost-utility of Etelcalcetide: a Markov model. Abstract presented at: ISPOR 19th Annual European Congress; October 31, 2016; Vienna, Austria.

92. Stollenwerk B, Iannazzo S, Cooper K, Belozeroff V. Exploring the potential value of improved care for secondary hyperparathyroidism (SHPT) with a novel investigational calcimimetics therapy. Abstract presented at: ISPOR 19th Annual European Congress; October 31, 2016; Vienna, Austria.

93. Gincherman Y, Moloney K, McKee C, Coyne DW. Assessment of adherence to cinacalcet by prescription refill rates in hemodialysis patients. Hemodial Int. 2010;14:68-72.
Therapeutics and Clinical Risk Management

\section{Publish your work in this journal}

Therapeutics and Clinical Risk Management is an international, peerreviewed journal of clinical therapeutics and risk management, focusing on concise rapid reporting of clinical studies in all therapeutic areas, outcomes, safety, and programs for the effective, safe, and sustained use of medicines. This journal is indexed on PubMed Central, CAS,

\section{Dovepress}

EMBase, Scopus and the Elsevier Bibliographic databases. The manuscript management system is completely online and includes a very quick and fair peer-review system, which is all easy to use. Visit http://www.dovepress.com/testimonials.php to read real quotes from published authors. 